\title{
Measuring the Effects of Ambient Gas Environment on Catalyst Defaceting and Coarsening through Environmental TEM
}

\author{
Sammy M. Saber ${ }^{1}$, Rahul Rao ${ }^{2}$, Seung Min Kim³ Avetik Harutyunyan ${ }^{2}$, and Eric A. Stach ${ }^{4}$
}

1. School of Materials Engineering and Birck Nanotechnology Center, Purdue University, West Lafayette, IN 47906 USA

2. Honda Research Institute, Columbus, OH 43212 USA

3. Korea Institute of Science and Technology, Seoul, Republic of Korea

4. Center for Functional Nanomaterials, Brookhaven National Laboratory, Upton, NY 11973 USA

Control of carbon nanotube (CNT) chirality during growth is of utmost importance for fabricating large numbers of CNTs with similar electrical properties. The term "chirality" refers to the structure of a CNT and determines whether it behaves as a semiconductor or a metal in terms of electrical properties. Current techniques used to grow CNTs can only grow both types of tubes. Recent work has shown a relationship between the ambient gas environment during growth and the resulting chirality of the CNTs [1]. In this study, it was found that in the presence of a $\mathrm{He} / \mathrm{H}_{2} \mathrm{O}$ ambient, the Fe nanoparticles used to nucleate CNTs maintained strong facets, while in the presence of an $\mathrm{Ar} / \mathrm{H}_{2} \mathrm{O}$ ambient they underwent a roughening transition. The strongly faceted particles were found to lead to preferential selection of metallic tubes. The origin of this phenomenon remains unclear. One hypothesis is that the $\mathrm{H}_{2} \mathrm{O}$ environment leads to $\mathrm{OH}$ molecules being preferentially adsorbed to the Fe nanoparticles: in the He environment, there is insufficient transfer of energy to the $\mathrm{OH}$ molecules to lead to their desorption while in the Ar environment, the greater mass of the incident gas molecules may knock the $\mathrm{H}_{2} \mathrm{O}$ off the particles, leading to the observed defaceting. We have designed a series of experiments to investigate the cause of this phenomenon by comparing ambient gasses by their Knudsen number, a dimensionless constant relating a molecule's size to its mean-free-path.

To test the ability of gases to transfer their momentum to catalyst particles, we utilized chemical vapor deposition and in-situ transmission electron microscopy techniques (TEM). . Each study was performed in different ambient gas environments (helium, neon, argon, and xenon) both with and without water. In addition, the pressure of each ambient gas was scaled to normalize its Knudsen number. These samples were then characterized inside of a TEM and then correlated with in-situ TEM studies. Selected experiments were repeated in-situ in an environmental transmission electron microscope, to dynamically observe these same changes. Correlating the faceting changes in each gas environment leads to a general model describing how ambient gasses effect catalyst morphology.

\section{References}

1. Harutyunyan, A.R., et al., Preferential Growth of Single-Walled Carbon Nanotubes with Metallic Conductivity. Science, 2009. 326: p. 116-120.

2. Work at Purdue University supported by the Honda Research Institute. EAS acknowledges funding to the Center for Functional Nanomaterials, Brookhaven National Laboratory, which is supported by the U.S. Department of Energy, Office of Basic Energy Sciences, under Contract No. DE-AC02-98CH10886 

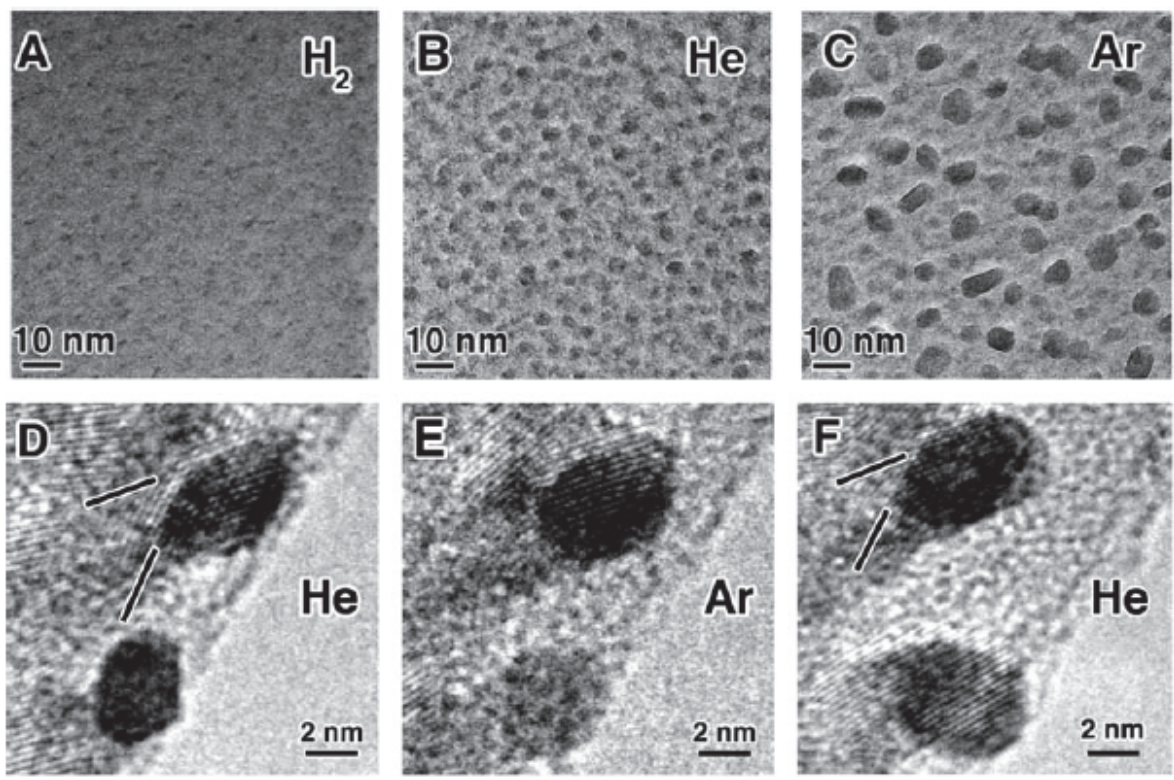

Figure 1. Plan-view TEM images of iron catalyst nanoparticle evolution after annealing in (A) $\mathrm{H}_{2}$, (B) He, and (C) Ar for 60 min in the presence of water. A clear difference in Ostwald ripening rates can be observed between the three gases. Figures (D), (E), and (F) are high resolution TEM images of the same two iron nanoparticles being observed in water as ambient gas environment changes from $\mathrm{He}$ to $\mathrm{Ar}$ and back to He, respectively. It is observed that the nanoparticles first exhibit strong facets, but then begin to round as Ar is introduced. These facets are then renewed as the gas environment changes back to $\mathrm{He}[1]$.
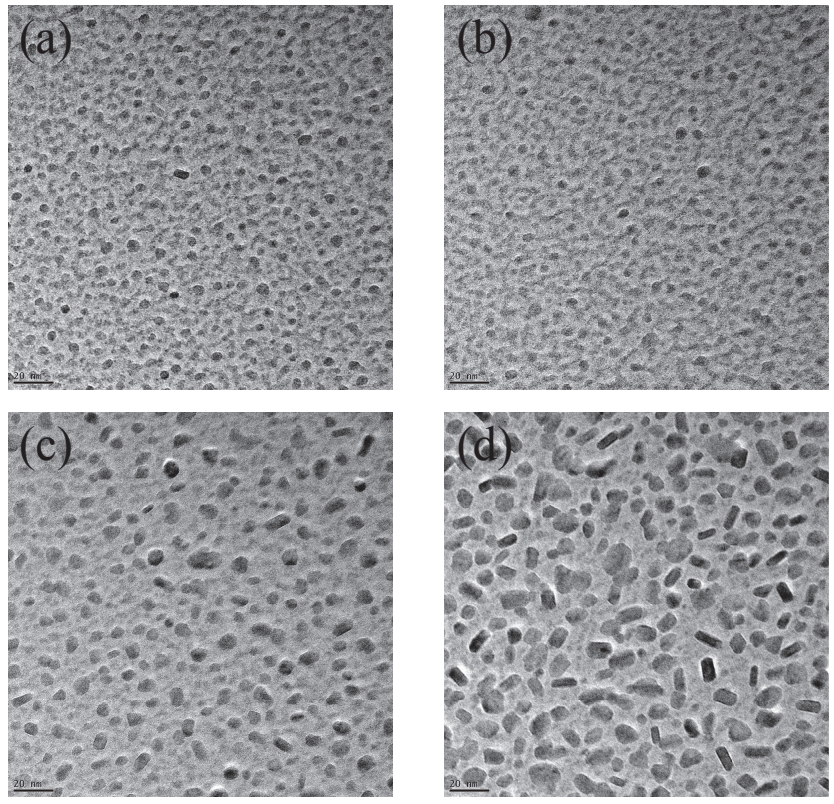

Figure 2. Plan view TEM images of iron catalyst particles annealed at $500^{\circ} \mathrm{C}$ with water for $30 \mathrm{~min}$ in (a) 1.5 mTorr, (b) 20 mTorr, (c) 500 mTorr, and (d) 1200 mTorr of Ar. By scaling the Knudsen number of the Ar gas by increasing its pressure, we observe increased Ostwald ripening rates as the Knudsen number of the gas increases. 